\title{
Ein Lebenswerk für die zweite Revolution in der Medizin
}

Peter Weibel

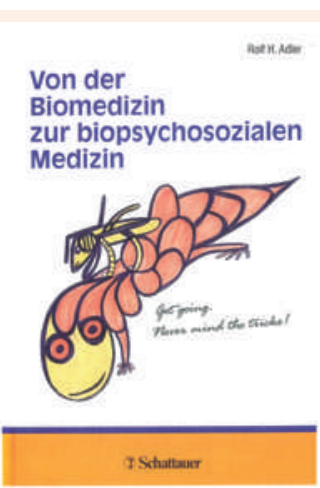

Rolf Adler

Von der Biomedizin zur biopsychosozialen Medizin Stuttgart: Schattauer; 2013. 154 Seiten. $40.90 \mathrm{CHF}$ ISBN 978-3-7945-3044-1

Korrespondenz: Peter Weibel Tilia Stiftung für Langzeitpflege Jupiterstrasse 65 CH-3000 Bern 15 Tel. 0319406111

peter.weibel[at]tilia-stiftung.ch
Er ist ein wunderbarer Erzähler, einige wissen es schon, viele werden es erfahren, wenn sie dieses Buch gelesen haben. Rolf Adler hat als Professor für psychosomatische Medizin und Chefarzt des Loryspitals in Bern die Fundamente für das biopsychosoziale Denken und Handeln in der Medizin gelegt. In diesem Buch erzählt er seine Lebensgeschichte, und seine Lebensgeschichte erzählt von fünfzig Jahren Medizingeschichte: Sein Leben - von der Geschichte der Medizin geprägt - und die neuen Dimensionen in der Medizin - von seinem Leben geprägt.

Die neuen Dimensionen in der Medizin sind grenzüberschreitend. So einleuchtend, so unwiderlegbar sie jedem erscheinen, so unfassbar muten die Widerstände an, denen Rolf Adler während Jahrzehnten ausgesetzt war: die Überwindung einer Medizin für Körper ohne Seelen und einer für Seelen ohne Körper in einer Seelen-Körper-Medizin: im biopsychosozialen Verstehensmodell. Rolf Adler hat dafür gekämpft, er wurde dafür ausgegrenzt, aber mit seiner Überzeugung und seinem Charisma hat er Meilensteine gesetzt und Ärztinnen und Ärzte geprägt, die seine Überzeugung weitertragen; es sind Hunderte.

So schreibt er über seine Kämpfe, seine Enttäuschungen - und über die späte Genugtuung. «Wenn man aus tiefer Überzeugung einen eigenen Weg verfolgt, weiss man nicht, wie weit er führen wird, aber es ist richtig, ihn zu gehen.» Rolf Adler schreibt packend, mal leise lächelnd, mal streitbar, oft in wunderbaren Anekdoten, immer sehr persönlich und immer selbstkritisch. Er gibt in diesem Buch preis, wer er ist, wer er auch ist, wer man sein muss, um die zweite Revolution in der Medizin, den Paradigmenwechsel von der mechanistischen zur biopsychosozialen Medizin voranzutreiben. Denn man muss vieles gleichzeitig sein, gleichzeitig verstehen, um eine zusammenhängende Wirklichkeit zu erkennen. Erst der Ausbruch aus der Einseitigkeit, erst die Verknüpfung schaffen das Nochnicht-Gewordene. Und Rolf Adler ist vieles gleichzeitig: der geschulte Internist. Der Psychoanalytiker. Der akribische Wissenschaftler. Der Geschichts-Bewusste und Literaturkenner. Der charismatische Lehrer. Und immer und vor allem: der Mensch mit Herz, der sich über Herzlosigkeit und Vereinfachung empören kann und aus Empörung die Medizin revoltiert: Der Weg der reduktionistischen Körpermedizin reicht nicht. «Eine Diagnose ist wie die nach oben gerichtete Fläche eines Würfels, bei deren Betrachtung die anderen Flächen ausser Acht gelassen werden. Man darf nicht vergessen, dass der Würfel noch fünf weitere Flächen besitzt, die psychische und soziale Probleme beinhalten.»
Warum die vereinfachende Körpermedizin nicht reicht, belegt Rolf Adler an exemplarischen, präzise beschriebenen Patienten-Beispielen und an einem Gesprächsprotokoll, das für jeden fassbar ist. Das Körpersymptom ist die Spitze des Eisbergs, aber die vier Fünftel des Eisbergs unter der Oberfläche sind die unsichtbare Wirklichkeit, sie ist nur dem zugänglich, der die verborgenen Zeichen erkennen und deuten kann. Auch darüber, was geschehen kann, wenn die verborgene Wirklichkeit nicht erkannt oder missachtet wird, erzählen die beschriebenen Situationen in diesem Buch.

Jedes grosse Lebenswerk ist eingebettet in ein Kontinuum - ins Davor und Danach. In prägenden Einflüssen, die das Lebenswerk vorbereitet haben und in geprägten Beeinflussten, die es weiterführen. Das Kontinuum ist ein zusammenhängendes Netzwerk von Gleichgesinnten, von Persönlichkeiten, von Lehrern und Schülern, die mittragen und getragen werden. Rolf Adler gibt ihnen Raum und Wort in seinem Buch: Briefwechsel als Landkarten von Verbindungen, die eine gemeinsame Überzeugung geschaffen hat - mit George L. Engel in Rochester und Thure von Uexküll in Ulm. Der Dank an die Pioniere der biopsychosozialen Medizin und der Dank der Schweizer Ärzte und Ärztinnen an den Berner Pionier der biopsychosozialen Medizin: Sie sind inzwischen Allgemeinärzte, Psycho-Onkologinnen und selbst Chefärzte geworden.

Kommt das Buch zur richtigen oder zur falschen Zeit? Vielleicht ist die falsche Zeit die richtige Zeit die psychosomatische Abteilung des Loryspitals ist im Herbst 2013 geschlossen worden. Vorläufig? Die Notwendigkeit einer integrierten biopsychosozialen Medizin, die den Körper mit der Seele und die Seele mit dem Körper zu behandeln versteht, ist heute unantastbar geworden: Rolf Adler hat das Fundament für ihre Bedeutung gelegt. Und es sind nicht mehr die anderen Fachärzte, die ihre Bedeutung in Frage stellen, die sie beschneiden, es sind die medizinischen Funktionäre und Reissbrett-Ökonomen mit ihrer vorgetäuschten Kostenwahrheit. Weder Akutklinik noch Psychiatrie werden Patienten mit langdauernden, quälenden Schmerzen oder schweren Essstörungen gegenüber gerecht werden können. Die Empörung darüber müsste gross sein, aber sie ist noch nicht gross genug. So müsste Rolf Adlers packendes, vielschichtiges Buch über sein Leben und über die zweite Revolution in der Medizin, der schon wieder Gegenkräfte entgegengesetzt werden, nicht nur faszinieren. Es müsste aufrütteln. 